\title{
Arabidopsis thaliana population analysis reveals high plasticity of the genomic region spanning $M S H 2$, AT3G18530 and AT3G18535 genes and provides evidence for NAHR-driven recurrent CNV events occurring in this location
}

Agnieszka Zmienko ${ }^{1,2}$, Anna Samelak-Czajka², Piotr Kozlowski ${ }^{1}$, Maja Szymanska ${ }^{1}$ and Marek Figlerowicz ${ }^{1,2^{*}}$

\begin{abstract}
Background: Intraspecies copy number variations (CNVs), defined as unbalanced structural variations of specific genomic loci, $\geq 1 \mathrm{~kb}$ in size, are present in the genomes of animals and plants. A growing number of examples indicate that CNVs may have functional significance and contribute to phenotypic diversity. In the model plant Arabidopsis thaliana at least several hundred protein-coding genes might display CNV; however, locus-specific genotyping studies in this plant have not been conducted.

Results: We analyzed the natural CNVs in the region overlapping MSH2 gene that encodes the DNA mismatch repair protein, and AT3G18530 and AT3G18535 genes that encode poorly characterized proteins. By applying multiplex ligation-dependent probe amplification and droplet digital PCR we genotyped those genes in $189 \mathrm{~A}$. thaliana accessions. We found that AT3G18530 and AT3G18535 were duplicated (2-14 times) in 20 and deleted in 101 accessions. MSH2 was duplicated in 12 accessions (up to 12-14 copies) but never deleted. In all but one case, the MSH2 duplications were associated with those of AT3G18530 and AT3G18535. Considering the structure of the CNVs, we distinguished 5 genotypes for this region, determined their frequency and geographical distribution. We defined the CNV breakpoints in 35 accessions with AT3G18530 and AT3G18535 deletions and tandem duplications and showed that they were reciprocal events, resulting from non-allelic homologous recombination between 99 \%identical sequences flanking these genes. The widespread geographical distribution of the deletions supported by the SNP and linkage disequilibrium analyses of the genomic sequence confirmed the recurrent nature of this CNV. (Continued on next page)
\end{abstract}

\footnotetext{
* Correspondence: marekf@ibch.poznan.pl

${ }^{1}$ Institute of Bioorganic Chemistry, Polish Academy of Sciences,

Noskowskiego 12/14, 61-704 Poznan, Poland

${ }^{2}$ Institute of Computing Science, Poznan University of Technology, Piotrowo

2, 60-965 Poznan, Poland
} 
(Continued from previous page)

Conclusions: We characterized in detail for the first time the complex multiallelic CNV in Arabidopsis genome. The region encoding MSH2, AT3G18530 and AT3G18535 genes shows enormous variation of copy numbers among natural ecotypes, being a remarkable example of high Arabidopsis genome plasticity. We provided the molecular insight into the mechanism underlying the recurrent nature of AT3G18530-AT3G18535 duplications/deletions. We also performed the first direct comparison of the two leading experimental methods, suitable for assessing the DNA copy number status. Our comprehensive case study provides foundation information for further analyses of CNV evolution in Arabidopsis and other plants, and their possible use in plant breeding.

Keywords: Copy number variation (CNV), Multiallelic CNV, Non-allelic homologous recombination (NAHR), Recurrent deletion, Multiplex ligation-dependent probe amplification (MLPA), Droplet digital PCR, Genotyping, Arabidopsis thaliana

\section{Background}

The rapid improvement of high throughput sequencing methods and the consecutive boost in genomic studies over the past few years have revealed the unexpectedly wide extent of intraspecies structural variation in the genomes of both animals and plants. Copy number variation $(\mathrm{CNV})$ is a type of genetic polymorphism manifested through varying copy numbers of large genome fragments (typically more than $1 \mathrm{~kb}$ ) [1]. CNV regions often span protein coding genes [2-7]. Changes in the number of functional gene copies (or their distal regulatory regions) might affect the amount of expressed protein and consequently alter the phenotype. Indeed, recent reports have clearly shown that in humans, $\mathrm{CNV}$ substantially impacts genome evolution, phenotypic variation and adaptation [8-11]. Similarly, the importance of CNV for plant fitness has been vividly demonstrated, e.g., the rapid (within a decade) spreading of glyphosate resistance among American populations of the weed plant, palmer amaranth (Amaranthus palmeri), resulting from the amplification of the gene encoding 5enolpyruvylshikimate 3-phosphate (EPSP) synthase, which is targeted by this herbicide [12]. Additionally, increased resistance to soybean cyst nematode $(\mathrm{SCN})$ reported in some soybean (Glycine max) lines has been associated with the duplication of the genomic region $R h g 1$, which spans 3 genes likely involved in counteracting the pathogen infection [13]. Some maize (Zea mays) lines have superior tolerance to high aluminum ion concentration, reflecting the triplication of the MATE-1 gene, which encodes an anion transporter, while in barley (Hordeum vulgare), the amplification of the boron transporter gene Bot1 increased plant tolerance to boron toxicity $[14,15]$. Other examples of plant CNVs also concern crops and traits important for plant breeding [16]. CNV has been associated with differences in flowering time in several species, including wheat (Triticum aestivum), barley and rapeseed (Brassica napus) [17-21]. The rice (Oryza sativa) landrace Ping13 has a superior grain length and quality that reflects the tandem duplication of the GL7 gene, which encodes a protein homologous to Arabidopsis (Arabidopsis thaliana) LONGIFOLIA proteins [22].

These observations highlight the need for the systematic discovery and validation of genes that undergo $\mathrm{CNV}$ to characterize the mechanisms driving $\mathrm{CNV}$ formation in plants. To date, the molecular studies regarding this issue are scarce. In soybean, phylogenetic analysis revealed the common origin of $\mathrm{SCN}$ resistance from a single progenitor, followed by subsequent copy number expansion (tandem duplications) and the divergence of the Rhg1 locus [23]. The duplicated copies of the gene encoding EPSP synthase are spread throughout the genome of palmer amaranth; therefore, the involvement of transposable elements in $\mathrm{CNV}$ formation has been assumed [12]. In both cases, strong positive selection pressure contributed to the spread of gene duplications. Moreover, genetic studies have revealed multiple examples of recurrent $\mathrm{CNVs}$ present in human populations, primarily triggered through the genomic architecture. One mechanism shown to be involved in the formation of recurrent CNVs in humans is non-allelic homologous recombination (NAHR) occurring between regions (typically longer than $1 \mathrm{~kb}$ ) of high sequence homology (over $95 \%$ ), known as segmental duplications or low copy repeats (LCRs) [24, 25]. Considering the numerous largescale genome events, such as polyploidy, or duplications of large chromosomal regions in plants [26-28], NAHR might also be expected to significantly contribute to $\mathrm{CNV}$ formation in plants.

Based on preliminary data from the Arabidopsis 1001 Genomes Project, the draft map of CNVs in this model plant was generated [29]. This effort has been the only population-scale genome-wide CNV detection study in this plant published to date. The map was based on whole-genome sequencing (WGS) data of 80 natural accessions, originating from various geographic locations in Europe, Asia and North Africa (MPICao2010 set). Using the read depth analysis, the authors inferred 1,059 CNVs (1 - $13 \mathrm{~kb}$ long), covering $1.8 \%$ of the Arabidopsis reference genome and partially or completely spanning 
more than 500 protein-encoding genes. From this dataset, we selected a region, encompassing 3 genes ( $M S H 2 /$ AT3G18524, AT3G18530 and AT3G18535), where Cao et al. inferred high-level duplications and frequent deletions [29]. $M S H 2$ codes for an Arabidopsis homolog of the MutS protein, an essential component of the DNA mismatch repair system [30, 31], while AT3G18530 and AT3G18535 encode proteins with poor functional annotation. We analyzed the sequence of this genomic region in the reference genome (TAIR10) and observed that AT3G18530 and AT3G18535 are flanked by $1 \mathrm{~kb}$ long direct repeats of $99 \%$ identity which suggested the possible involvement of the NAHR mechanism in CNV formation. In contrary to human genome where numerous NAHRderived CNVs are under deep investigation [25, 32-34], we are not aware of similar studies performed in plants. Due to the high level of variation suggested by the WGS data [29] the region encompassing AT3G18530 and AT3G18535 genes could be therefore used as a great model to study NAHR in plants and to examine the influence of selection pressure on the evolution of a highly recombinogenic region. As a first step to evaluate this possibility, in the present study we calculated the copy numbers of MSH2, AT3G18530 and AT3G18535 genes in 189 Arabidopsis accessions. We analyzed the chromosome breakpoints in accessions with the AT3G18530 and AT3G18535 tandem duplications/deletions and we confirmed the involvement of the NAHR mechanism in the origin of these variations. By evaluating the geographical distribution of the identified genotypes, supported by the SNP analysis we revealed the recurrent nature of the analyzed CNV. Moreover, we directly compared the accuracy of two popular methods of locus-specific CNV analysis: multiplex ligation-dependent probe amplification (MLPA) [35] and droplet digital PCR (ddPCR) [36-38], which we used for the first time for CNV genotyping in plants. The present study is the first locus-specific analysis of a $\mathrm{CNV}$ region in Arabidopsis, providing insight into the mechanisms and factors driving CNV evolution and describing the successful adaptation of popular genotyping tools for plant genome research.

\section{Results}

MSH2, AT3G18530 and AT3G18535 genes undergo CNV in Arabidopsis population

According to Cao et al. [29], the AT3G18530 and AT3G18535 genes and a part of the MSH2 gene are covered by two distinct CNVs (CNV_611 and CNV_610) separated by a 1.5 -kb distance (Fig. 1). The two CNVs differed according to the frequency of copy number alterations (multiple instances of duplication/deletion at CNV_611 and only 4 accessions with duplications at CNV_610). In addition, we observed some interesting interrelatedness - the duplication at CNV_610 was accompanied by similar levels of duplication at CNV_611 (Additional file 1: Figure S1). We analyzed the DNA copy number in this region, focusing on the protein-coding genes. The analysis comprised a set of 189 Arabidopsis accessions from the Arabidopsis 1001 Genomes Project [39]. Our experimental set included all 80 accessions represented in the MPICao2010 set and 109 accessions from the Salk collection. As a control and a calibrator with 2 copies per diploid genome of each analyzed gene, we used Col-0, which also served as a reference accession in the WGS-based CNV genotyping study [29].

MLPA is one of the most widely used methods of CNV genotyping in humans. In MLPA, each individual probe is divided into two fragments (half-probes), which are ligated and amplified only if these fragments hybridize to the analyzed region. The amount of the amplified product, determined through capillary electrophoresis, reflects the dosage of the DNA template. MLPA assays can be multiplexed after designing probes that generate products of a unique length, visible as separate peaks on electropherograms. We have previously optimized an approach based on synthetic oligonucleotides for the easy design of MLPA probes for genotyping numerous human CNVs [40, 41]. Here, we applied this method to design the Ath_CNV610611_MLPA probe set to target the genes of interest (Fig. 1 and Additional file 1: Figure S2). Two MLPA probes were designed for each gene: $\mathrm{mlpaB}$ and $\mathrm{mlpaC}$ for $M S H 2$, mlpaD and mlpaE for AT3G18530, and $\mathrm{mlpaF}$ and mlpaG for AT3G18535. For MSH2, one probe from the pair was located within CNV_610 (which overlaps exons 3-6), while the second probe was located in exon 11, outside of the predicted CNV. Additional probes were designed for genes located outside the inferred CNVs, flanking the variable DNA segment: mlpaA for HDA15 (AT3G18520) and mlpaH for BRC1 (AT3G18550). Altogether, the Ath_CNV610611_MLPA assay covered $25.5 \mathrm{~kb}$ of the Arabidopsis genome. We also designed 5 probes, each targeting one protein-coding gene with stable copy numbers (according to WGS analysis) located on different chromosomes. The respective probes (ctrl1-ctrl5) were generated to cover the entire range of DNA fragment lengths amplified in the Ath_CNV610-611_MLPA multiplex assay (96-172 bp) and served as internal controls (Table 1).

Each MLPA probe in this assay produced a well-resolved peak at the expected position (migration rate) in the electropherograms (Additional file 1: Figure S3). Changes in the gene copy number within the analyzed set of accessions were detected through the comparison of the normalized peak heights for each probe (Fig. 2). As expected, no CNV was observed for the HDA15 and BRC1 genes and the regions selected as controls in any accession. In 67 accessions, 


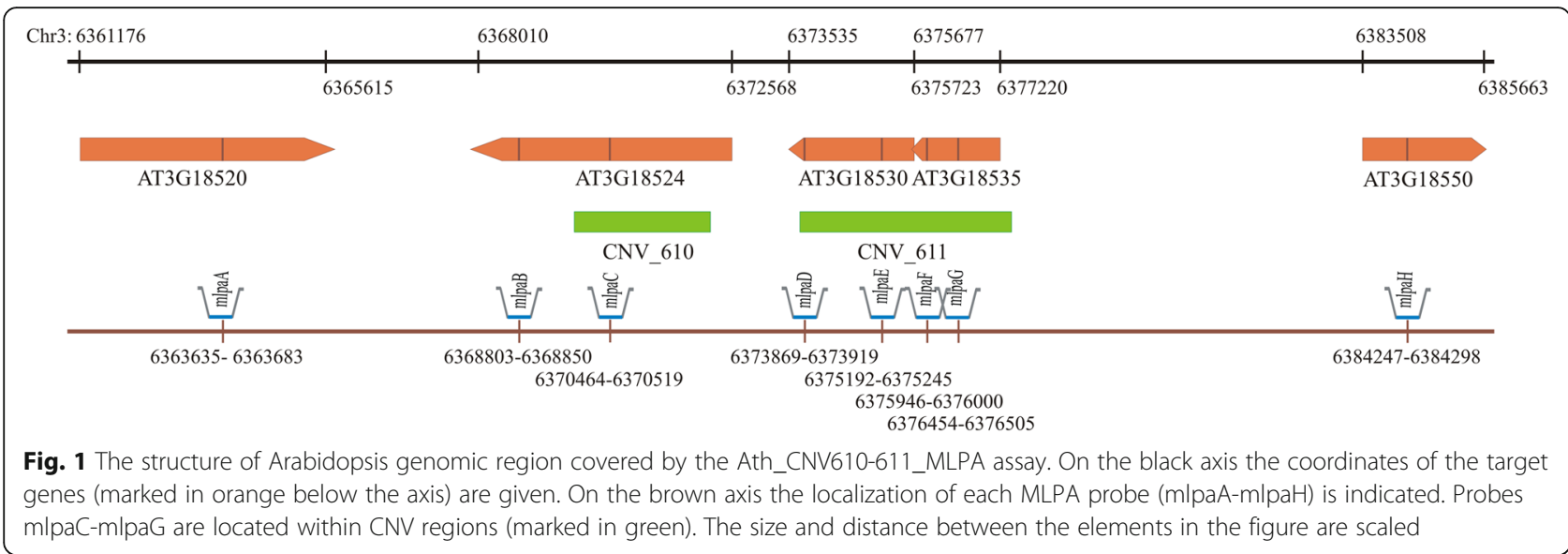

the copy numbers for MSH2, AT3G18530 and AT3G18535 genes were similar to those detected in Col-0; therefore, we referred to this genotype as "basic". In these samples, all probes (mlpaA-mlpaH, ctrl1-ctrl5) presented low signal variations $(\mathrm{SD}<10 \%)$, indicative of reliable and consistent performance [42]. As many as 101 accessions (53.4 \%) harbored deletions of AT3G18530 and AT3G18535 (genotype "del-2"). Duplications of at least one gene were detected in

Table 1 Probes used in the Ath_CNV610-611_MLPA assay and their target genes

\begin{tabular}{|c|c|c|c|}
\hline Probe & Locus & Gene product & $\begin{array}{l}\text { Amplified } \\
\text { fragment } \\
\text { length (bp) }\end{array}$ \\
\hline $\mathrm{mlpaA}$ & AT3G18520 & $\begin{array}{l}\text { HDA15, protein with similarity to } \\
\text { histone deacetylases }\end{array}$ & 108 \\
\hline mlpaB & \multirow[t]{2}{*}{ AT3G18524 } & \multirow{2}{*}{$\begin{array}{l}\text { MSH2, MutS protein homolog 2, } \\
\text { involved in maintaining genome } \\
\text { stability and repressing } \\
\text { recombination of mismatched } \\
\text { heteroduplexes }\end{array}$} & 152 \\
\hline $\mathrm{mlpaC}$ & & & 128 \\
\hline mlpaD & \multirow[t]{2}{*}{ AT3G18530 } & \multirow[t]{2}{*}{ ARM repeat superfamily protein } & 168 \\
\hline mlpaE & & & 117 \\
\hline $\mathrm{mlpaF}$ & \multirow[t]{2}{*}{ AT3G18535 } & \multirow[t]{2}{*}{ Tubulin-tyrosine ligase } & 160 \\
\hline mlpaG & & & 99 \\
\hline $\mathrm{mlpaH}$ & AT3G18550 & $\begin{array}{l}\text { BRC1, a TCP transcription factor, } \\
\text { arrests axillary bud development } \\
\text { and prevents axillary bud } \\
\text { outgrowth }\end{array}$ & 136 \\
\hline ctrl1 & AT1G01040 & $\begin{array}{l}\text { DCL1, a RNA helicase involved in } \\
\text { microRNA processing }\end{array}$ & 96 \\
\hline ctrl2 & AT4G21580 & $\begin{array}{l}\text { oxidoreductase, a zinc-binding } \\
\text { dehydrogenase family protein }\end{array}$ & 111 \\
\hline $\mathrm{ctrl3}$ & AT2G36230 & $\begin{array}{l}\text { APG10, a BBMII isomerase } \\
\text { involved in histidine biosynthesis }\end{array}$ & 124 \\
\hline $\mathrm{ctrl} 4$ & AT5G23290 & $\begin{array}{l}\text { PFD5, a prefoldin involved in } \\
\text { unfolded protein binding }\end{array}$ & 144 \\
\hline ctrl5 & AT1G73010 & $\begin{array}{l}\text { PS2, a pyrophosphate-specific } \\
\text { phosphatase }\end{array}$ & 172 \\
\hline
\end{tabular}

the remaining accessions, and several duplication patterns were observed. In 1 accession, $M S H 2$ was the only duplicated gene (genotype "dupl-1"). In 9 accessions, AT3G18530 and AT3G18535 genes were duplicated (genotype "dupl-2"). In 6 accessions, MSH2, AT3G18530 and AT3G18535 genes were duplicated (genotype "dupl-3-a"). In the remaining 5 accessions, the duplication of all 3 genes was also observed, but the signal from probe mlpaD was markedly higher than the signals from other probes (genotype "dupl-3-b"). Regardless of the latter, the signal correlations of MLPA probe pairs specific for particular genes were high, as were the pairwise signal correlations of all 4 probes covering the block of AT3G18530 and AT3G18535 genes (Additional file 1: Figure S4). A higher divergence of the outermost probes mlpaD and mlpaG might reflect the variations observed in the sequence and/ or location of $\mathrm{CNV}$ breakpoints among the individual DNA copies, thereby affecting MLPA probe hybridization. Thus, we concluded that AT3G18530 and AT3G18535 overlap a single CNV, and we inferred the same copy number genotypes for both genes in all analyzed accessions using mlpaE and mlpaF probes (see below).

\section{Copy numbers of MSH2, AT3G18530 and AT3G18535 genes in Arabidopsis population}

In previous reports, we showed that the copy numbers of the individual genes could be accurately determined after analyzing the data obtained for two independent MLPA probes on two-dimensional plots [41, 43]. Such plots were generated for the probes targeting $\mathrm{MSH} 2$ (mlpaB-mlpaC) and the AT3G18530-AT3G18535 block (mlpaE-mlpaF). The results showed that clusters of accessions with different copy numbers could be easily distinguished (Fig. 3). As Arabidopsis is primarily selfpollinating and laboratory-maintained plants are typically homozygous, we assumed that subsequent clusters corresponded to genotypes differing by two copies. For $M S H 2$, we distinguished 4 clusters with the 


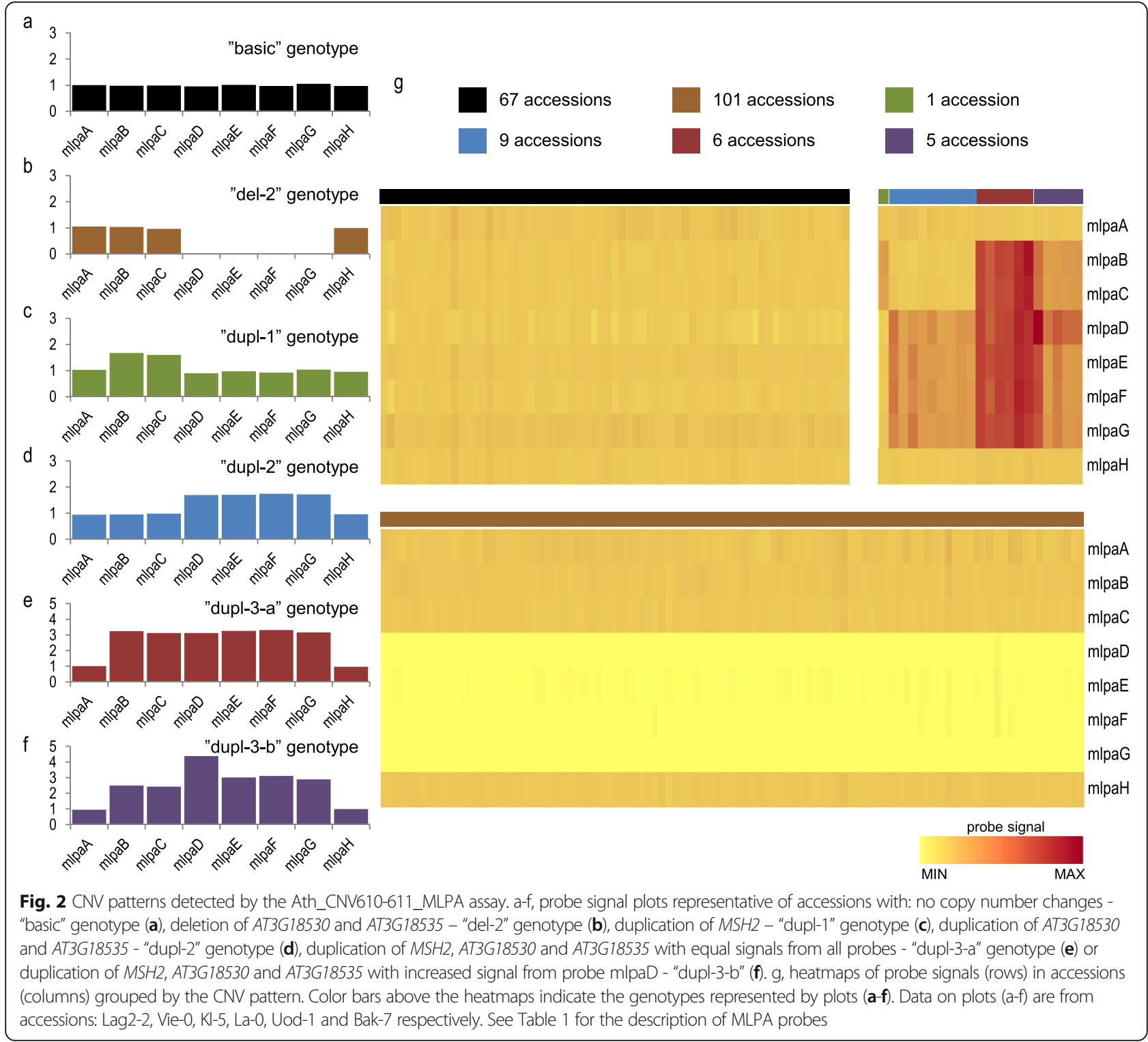

following copy numbers: 2 gene copies (in 177 accessions), 4 copies (in 5 accessions), 6 copies (in 2 accessions) and at least 8 copies (in 5 accessions). For AT3G18530 and AT3G18535, we observed 5 clusters. The apparent lack of a signal from both probes indicated gene deletion in 101 accessions. In 68 accessions, 2 copies were detected. In the remaining cases, duplications were observed: 4 copies in 10 accessions, 6 copies in 3 accessions and at least 8 copies in 7 accessions.

For MLPA assays, the distance between the clusters decreases with increasing integer copy number [41]. Therefore, we did not attempt to partition the relatively small clusters of accessions containing 8 or more copies of MSH2 and AT3G18530-AT3G18535 genes. To calculate the number of gene copies in the accessions with the highest level of duplications, we applied ddPCR. In this approach, the DNA template is highly diluted, facilitating the amplification of single copies of the target region, distributed among thousands of independent reaction partitions (droplets). After applying Poisson statistics, the copies of targeted DNA are quantified after counting the positive (amplification signal detected) and negative (no signal) reaction droplets. In the present study, we used gene-specific primers to analyze all 21 accessions with "dupl-1", "dupl-2", "dupl-3-a" and "dupl-3b" genotypes, 48 randomly selected accessions with the "del-2" genotype and 23 randomly selected accessions with the "basic" genotype. The calculated gene copy numbers were compared with the MLPA data. Both methods were entirely consistent in the identification of copy number variation (duplication/deletion/no change). 

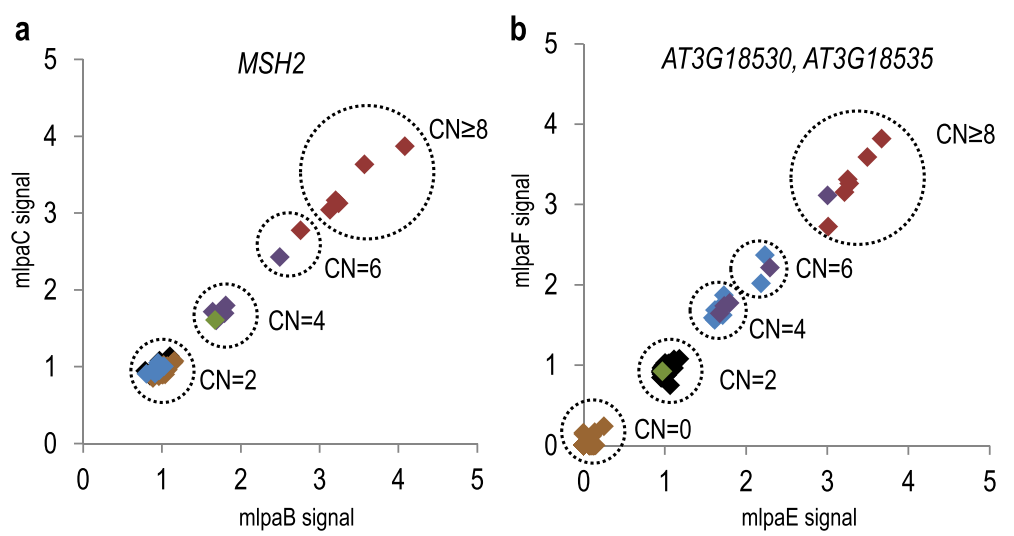

Fig. 3 Clusters of Arabidopsis accessions containing different gene copy numbers identified with Ath_CNV610-611_MLPA assay. The scatterplots present signals of paired MLPA probes for: a MSH2 and $\mathbf{b}$ AT3G18530-AT3G18535. All results were calibrated using data obtained for Col-0 accession. The clustering and copy number (CN) assignment was done manually. The data points (accessions) are colored according to the CNV patterns described in Fig. 2

The precise ddPCR-calculated copy numbers and MLPA probe intensities were highly but nonlinearly correlated, clearly revealing that ddPCR has much better resolution for genotyping highly duplicated genes (Fig. 4). Accordingly, ddPCR facilitates a more accurate determination of the gene copy numbers in the 7 accessions that could not be precisely characterized with MLPA. The estimated gene copy number in those accessions ranged from 8 to $12-14$ copies.

\section{Geographic distribution of CNV genotypes}

The samples analyzed in the present study originated from various locations: 143 accessions from Europe, 31 accessions from Asia, 14 accessions from North
America and 1 accession from North Africa (Morocco) (Additional file 2: Table S1). The most frequent genotype, "del-2", was detected in accessions from all investigated geographic regions (Fig. 5). In contrast, "dupl-1", "dupl-2", "dupl-3-a" and "dupl-3-b" patterns were primarily spread across various parts of Europe. Single accessions with the "dupl-3-a" and "dupl-3-b" genotypes were also detected in North America and Western Asia, respectively. Non-European accessions constituted only $23.8 \%$ of the experimental set, and the duplications were associated with the least abundant genotypes, even across Europe. We therefore consider it highly likely that sampling additional nonEuropean accessions would reveal additional examples
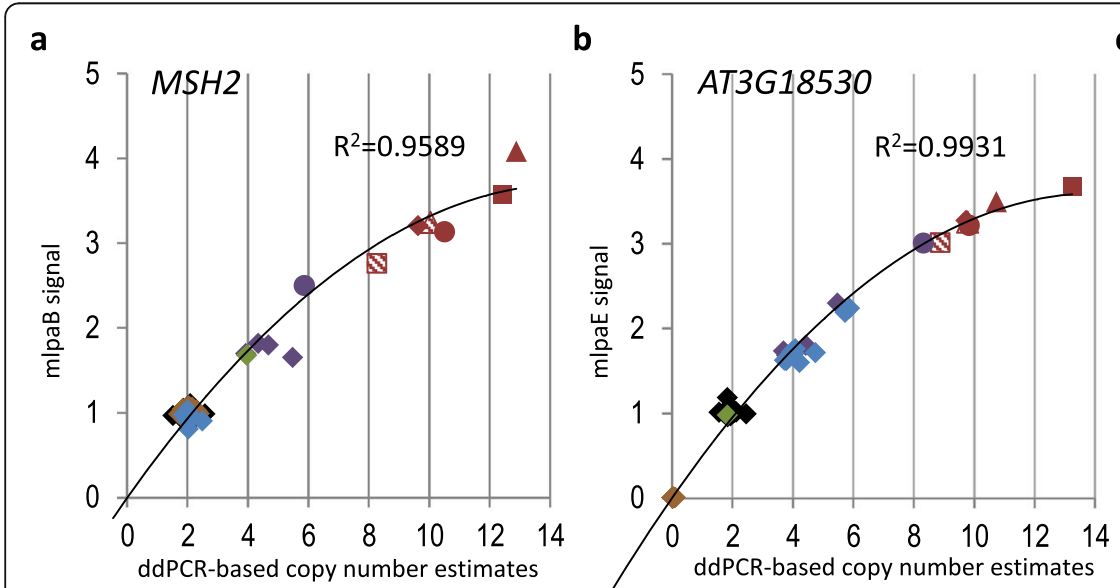

C

$\Delta$ Wei-0

Wal-HasB-4

Van-0

Uod-1

Ber

图 $\mathrm{Gr}-1$

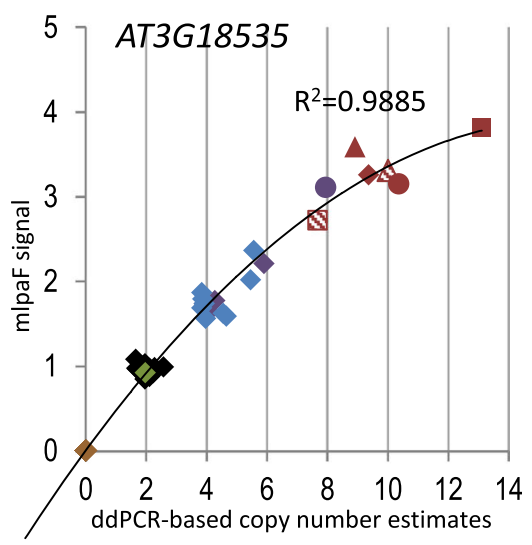

Fig. 4 Concordance of MLPA-based and ddPCR-based gene copy number genotyping results in 92 accessions. On x-asis, ddPCR-based absolute gene copy numbers are shown. On y-axis, normalized MLPA signals are shown, generated with the MLPA probe located nearest to the ddPCR primers' target position (Additional file 1: Figure S2). The data points (accessions) are colored according to the CNV patterns described in Fig. 2 and accessions with the highest levels of duplication are given unique symbols. Weaker data correlation observed for MSH2 in comparison with AT3G18530 and AT3G18535 is caused by the low number of accessions (12 out of 92 presented) with MSH2 gene copy number other than 2 


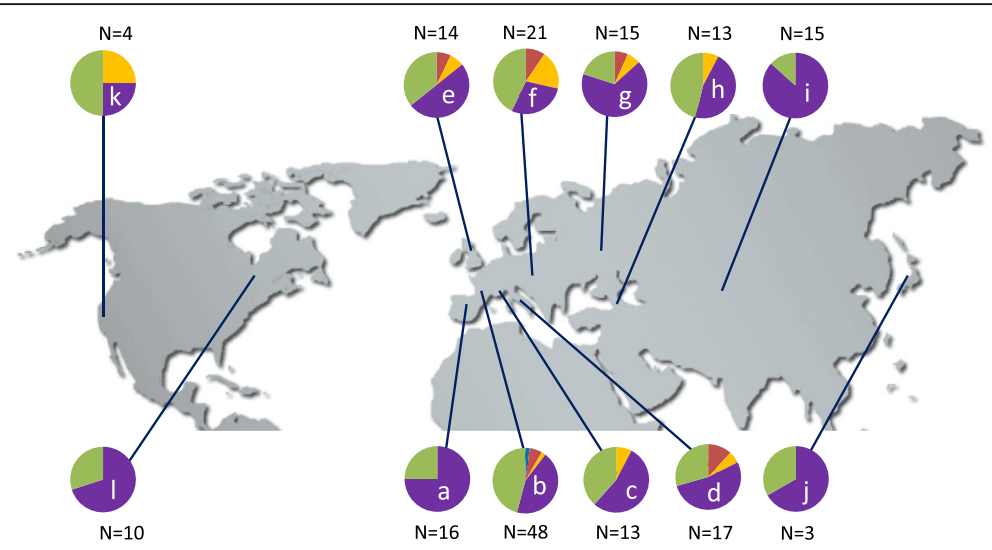

Fig. 5 Geographic distribution of the CNVs for the genomic region spanning MSH2, AT3G18530 and AT3G18535 genes. The number of accessions sampled for each region (N) is reported. Colors indicate genotypes: "basic" (green); "del-2" (purple), "dupl-1" (blue), "dupl-2" (red), "dupl-3-a" or

"dupl-3-b" (yellow). a - Iberian Peninsula \& Morocco, b - Western Europe, c - Alps, d - Italy, e - Northern Europe, f - Central \& Southeast Europe, g - Eastern Europe, h - Western Asia \& Caucasus, i - Central Asia, j - East Asia, k - Pacific Northwest, I - Midwest

of "dupl-1" and "dupl-2" genotypes within these groups. Although we did not observe any correlation between the genotype and geographic origin of the plants, to ascertain whether plants with particular CNV variants might originate from common ancestors, we analyzed the bi-allelic SNPs of at least $10 \%$ frequency in the 20-kb genomic regions flanking the investigated segment from both sides in a subset of 153 accessions for which well-validated SNP data has been released recently by 1001 Genomes Consortium [44] and additionally in Col-0. We constructed the phylogenetic network using the distance-based method NeighborNet, implemented in SplitsTree [45]. We then compared the distribution of the CNV genotypes with the genetic groups identified by wholegenome variation analysis of 1,135 Arabidopsis accessions [44]. The analysis did not reveal any clear evolutionary splits between accessions harboring distinct CNV genotypes (Additional file 1: Figure S5). One remarkably distinguishing network branch included accessions from Kyrgyzstan, Russia, Tajikistan, Uzbekistan, Armenia and USA, which all harbored "del-2" genotype. However, these Eurasian accessions were previously shown to present the lowest level of allele differentiation and the smallest number of private SNPs $[29,46]$. Also, according to recent phylogenetic data, the USA accessions belong to Germany genetic cluster and exhibit extensive haplotype sharing [44]. We did not detect the relationship between the CNV patterns and any other haplotypes (Fig. 6). We further analyzed the linkage disequilibrium (LD) of biallelic SNPs and CNV genotypes. Two regions of high LD were detected on both sides of the CNV segment, but similarly, no correlation between any SNP and any CNV pattern was observed $\left(\mathrm{R}^{2}<0.3\right)$ (Additional file 1: Figure S6). Altogether these observations supported the hypothesis that MSH2 and/or AT3G18530AT3G18535 copy number changes occurred independently in multiple accessions.

\section{Prevalence of "del-2" genotype in Arabidopsis population} The depth-of-coverage methods rely on counting the number of reads mapping to a particular genomic position to ascertain the DNA copy number and detect $\mathrm{CNV}$ regions. We analyzed the genomic sequence of AT3G18530-AT3G18535 loci in the recently released pseudogenome sequences for 1135 Arabidopsis accessions [44]. These pseudogenomes were generated based on WGS data, by combining reference and variant calls including indels, with uncalled sites represented as Ns. We observed that 687 accessions had at least $80 \%$ values missing in the analyzed region (Additional file 1: Figure S7). These included all accessions with "del-2" genotype, for which pseudogenomes were available (76 cases). On the contrary, none of the 18 accessions with the duplication genotypes and available pseudogenomes was found in this group. Likewise, only 2 out of 59 accessions with "basic" genotype had at least $80 \%$ missing values at this region. Thus, we concluded that the presence of the "del-2" genotype could be predicted with high efficiency and low false positive error from the missing values in the WGS data. Consequently, we estimated that the frequency of "del-2" genotype among 1,135 Arabidopsis accessions was $60.53 \%$. We further determined the "del-2" frequency in 10 countries with the highest sample representation in the above collection (Table 2). It ranged from $46.6 \%$ in Germany to $80.56 \%$ in Spain. Thus, we propose that the CNV of this genomic segment is widespread throughout the Arabidopsis population; in particular, AT3G18530 and AT3G18535 are frequently deleted. 


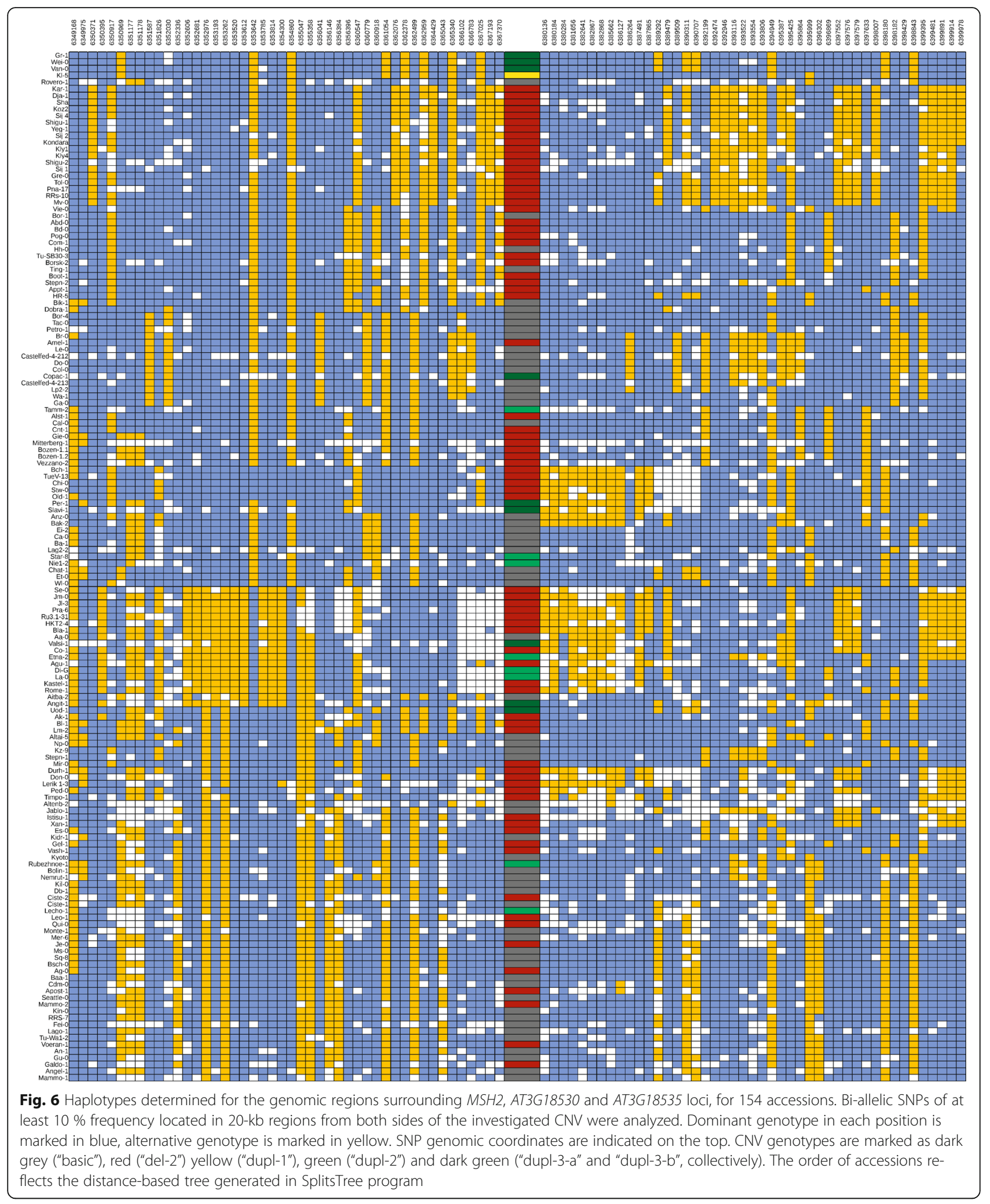


Sequence analysis of breakpoints in accessions with "del2 " and "dupl-2" genotypes

Our data indicated that AT3G18530 and AT3G18535 genes are located within a single CNV, and the individual genotypes are spread across various regions of the Arabidopsis habitat. Considering the high frequency of the "del-2" genotype in the global population, we concluded that the $\mathrm{CNV}$ spanning AT3G18530 and AT3G18535 genes might be recurrent. MSH2, AT3G18530 and AT3G18535 are all encoded on the minus strand of chromosome 3 . In the reference genome, two 1238-bp LCRs of $99 \%$ sequence identity flank the AT3G18530-AT3G1853 gene block. The left LCR separates MSH2 from AT3G18530, partially overlapping the first and last exon, of the former and latter gene, respectively. The right LCR is localized at $148 \mathrm{nt}$ upstream of the AT3G18535 gene start site (regarding the minus strand). Apart from those positions, the LCR sequence is not repeated elsewhere in the genome. We hypothesized that the natural variation of this region might be promoted through NAHR events, mediated through LCRs (Fig. 7a). According to the double strand break (DSB) repair model, intrachromatidal NAHR, involving a double Holliday junction $(\mathrm{dHj})$, might lead to the deletion of AT3G18530 and AT3G18535 genes, while interchromosomal or interchromatidal NAHR generates deletions and reciprocal tandem duplications [24, 32]. Using DNA sequencing, we confirmed that both LCRs were present in the expected

Table 2 Comparison of the frequency of "del-2" genotype across the countries, inferred from the analysis of Arabidopsis 1,001 Genomes Project WGS data and experimentally determined in current study

\begin{tabular}{|c|c|c|c|c|}
\hline \multirow[t]{2}{*}{ Country } & \multicolumn{2}{|c|}{$\begin{array}{l}\text { Number of } \\
\text { representing } \\
\text { accessions }\end{array}$} & \multicolumn{2}{|c|}{$\begin{array}{l}\text { Frequency of "del-2" } \\
\text { genotype }\end{array}$} \\
\hline & $\begin{array}{l}1,001 \\
\text { Genomes } \\
\text { Project }\end{array}$ & $\begin{array}{l}\text { This } \\
\text { study }\end{array}$ & $\begin{array}{l}\text { 1,001 } \\
\text { Genomes } \\
\text { Project }^{\mathrm{a}}\end{array}$ & $\begin{array}{l}\text { This } \\
\text { study }\end{array}$ \\
\hline All countries & 1135 & 189 & $60.61 \%$ & $53.44 \%$ \\
\hline $\begin{array}{l}\text { Highly represented } \\
\text { countries (listed below): }\end{array}$ & 979 & 128 & $64.04 \%$ & $56.25 \%$ \\
\hline Sweden & 243 & 1 & $55.97 \%$ & $0.00 \%$ \\
\hline Spain & 180 & 10 & $80.56 \%$ & $100.00 \%$ \\
\hline USA & 123 & 12 & $86.99 \%$ & $58.33 \%$ \\
\hline Germany & 118 & 32 & $46.61 \%$ & $37.50 \%$ \\
\hline Italy & 73 & 28 & $47.95 \%$ & $53.57 \%$ \\
\hline United Kingdom & 69 & 10 & $71.01 \%$ & $60.00 \%$ \\
\hline Russia & 60 & 16 & $80.00 \%$ & $75.00 \%$ \\
\hline France & 45 & 10 & $46.67 \%$ & $70.00 \%$ \\
\hline Czech Republic & 40 & 7 & $55.00 \%$ & $42.86 \%$ \\
\hline Bulgaria & 28 & 2 & $32.14 \%$ & $0.00 \%$ \\
\hline
\end{tabular}

aThe "del-2" genotype was assigned to accessions with at least $80 \%$ missing values in the sequence of the genomic region spanning AT3G18530 and AT3G18535 loci genomic positions in Col-0, and these sequences were identical to the reference genome. Subsequently, we amplified the deletion junction fragment in 27 accessions of various geographic origins harboring the "del-2" genotype. The lengths of the amplified products (approximately $3.4 \mathrm{~kb}$ in case of deletion versus approximately $8.4 \mathrm{~kb}$ in Col-0) were consistent with the above model, assuming the loss of a 5$\mathrm{kb}$ region including both genes and one LCR (Fig. $7 \mathrm{~b}$ ). Similarly, we performed breakpoint-spanning PCR to detect the tail-to-head orientation of the AT3G18535-AT3G18530 gene blocks with intervening LCR sequence in 8 lines with "dupl-2" genotypes. The presence of amplification products was confirmed for all accessions, although 3 of these products were longer (approximately $4.2 \mathrm{~kb}$ ) than predicted (approximately $3.4 \mathrm{~kb})(\mathrm{Fig} .7 \mathrm{c})$. No amplification product was observed in the case of Col- 0 , as expected.

The left and right LCRs differ by 11 nucleotides which divide this region of homology into 10 intervals and permit to map recombination breakpoints to short DNA stretches (Fig. 8). We partially sequenced the amplification products described above, confirming that the CNV breakpoint was indeed located within the LCR sequence. The switches between the sequences of the left and right LCRs in accessions with "del-2" genotype were localized between position 1 and 231 (the first interval), while the switches between the right and left LCR sequences in accessions with "dupl2 " genotype were mostly localized between position 231 and 769 (the second interval). The mutual arrangement between "del-2" and "dupl-2" genotypes accurately fits the NAHR model involving $\mathrm{dHj}$, that is subsequently resolved by crossover (see [33] for the description and schematic representation of the $\mathrm{dHj}$ formation and resolution). Therefore it supports the reciprocity of the duplication/deletion events leading to both genotypes. According to the above model, the DSB site is located between the two sequence junction sites detected in accessions with "del-2" and "dupl2 " genotypes, respectively. In this case the DSB site could be tracked to the 5 ' half of the LCR sequence (between position 1 and 769). This is the region of the longest uninterrupted homology between both LCRs (230 and 537 bp tracks, separated by only one mismatch, T/A). The track length is consistent with the range of minimal efficient processing segments (regions sharing extremely high similarity or identity necessary for NAHR to occur), as empirically estimated for human meiosis at 300 to 500 bp (reviewed in [24]). Additionally, in 7 accessions with the "del-2" genotype and in 5 accessions with the "dupl-2" genotype, the repeat sequence was a mixture of the left and right LCRs, reflecting discontinuous sequence conversion events [33]. In 3 accessions with duplications, the conversion track was extended approximately $790 \mathrm{bp}$ beyond the right LCR, resulting in larger duplication. The presence and length of this extended duplication were consistent with the size of the amplification products detected on the gel 


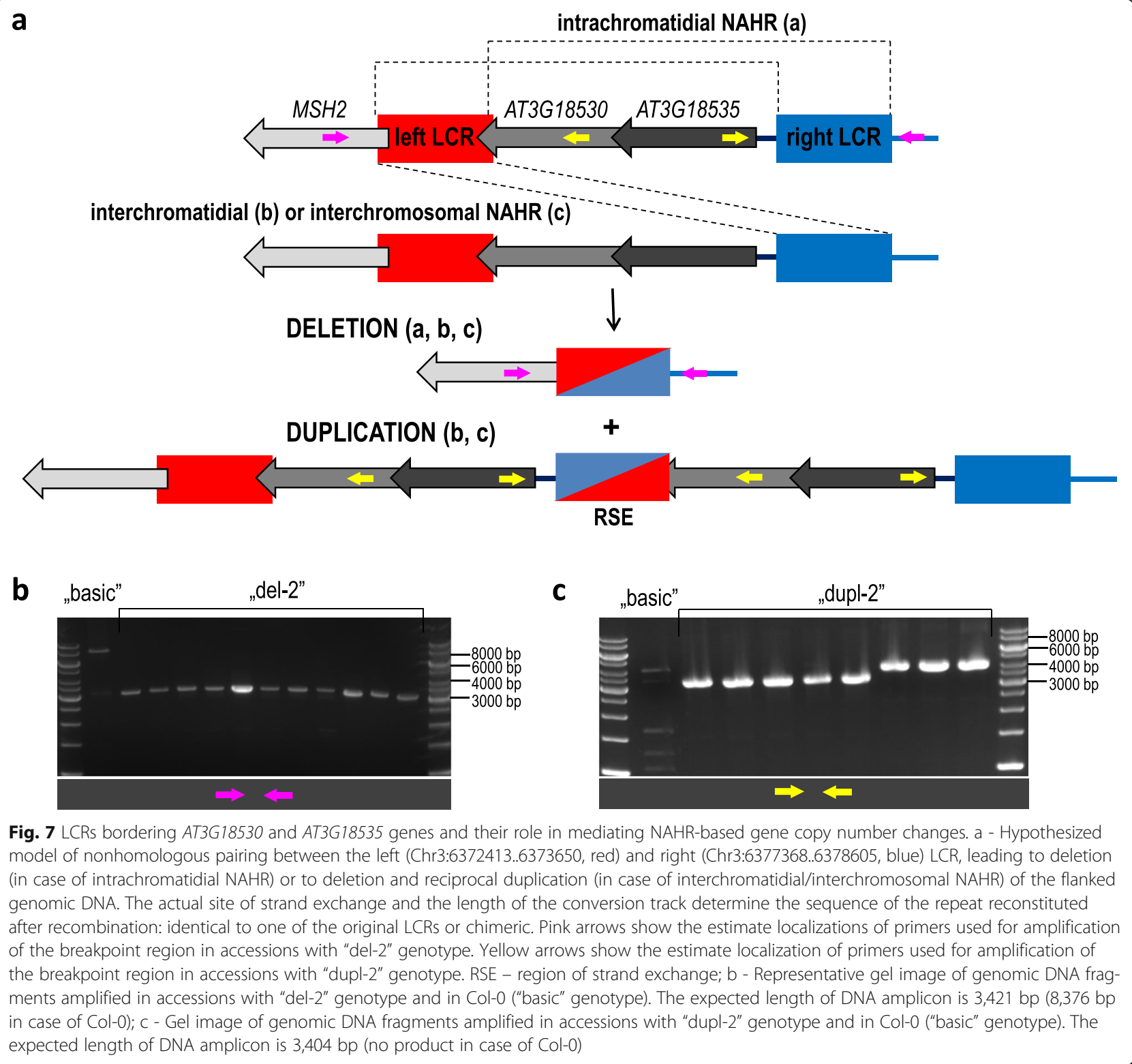

electropherogram presented above. Interestingly, the sequence restoration in those accessions was likely mediated through the 9-nt microhomology between the duplicated fragment and the 3' border of the left LCR (Additional file 1: Figures S8- S11). In addition, substantial sequence variation was observed around the breakpoint region, both in lines with "del-2" and with "dupl-2" genotype, consistent with previous findings for $\mathrm{CNV}$ in humans [47].

\section{Discussion}

The information derived from high-throughput sequencing projects revealed that $\mathrm{CNV}$ affects a substantial part of plant genomes [48-50]. However, in plants, the CNV maps or detailed characteristics of individual CNVs are not abundant. Most notably, only one genome-wide
$\mathrm{CNV}$ analysis has been performed in the top model plant Arabidopsis [29] and none of the identified loci have been studied in detail, apart from the brief verification of presence-absence variants [51]. Here, we presented the first detailed analysis of a complex CNV in the Arabidopsis genome with a wide range of changes in the copy number across individuals. This type of polymorphism is referred to as multiallelic CNV (mCNV). In humans, mCNVs have received much attention, reflecting the high prevalence of these changes in the genome and the fact that these variations frequently affect protein-coding genes. Examples of mCNVs that modify the human phenotype include the CCL3L1 and DEFB genes, which are associated with infectious and inflammatory-related diseases [52]. Additionally, it was 


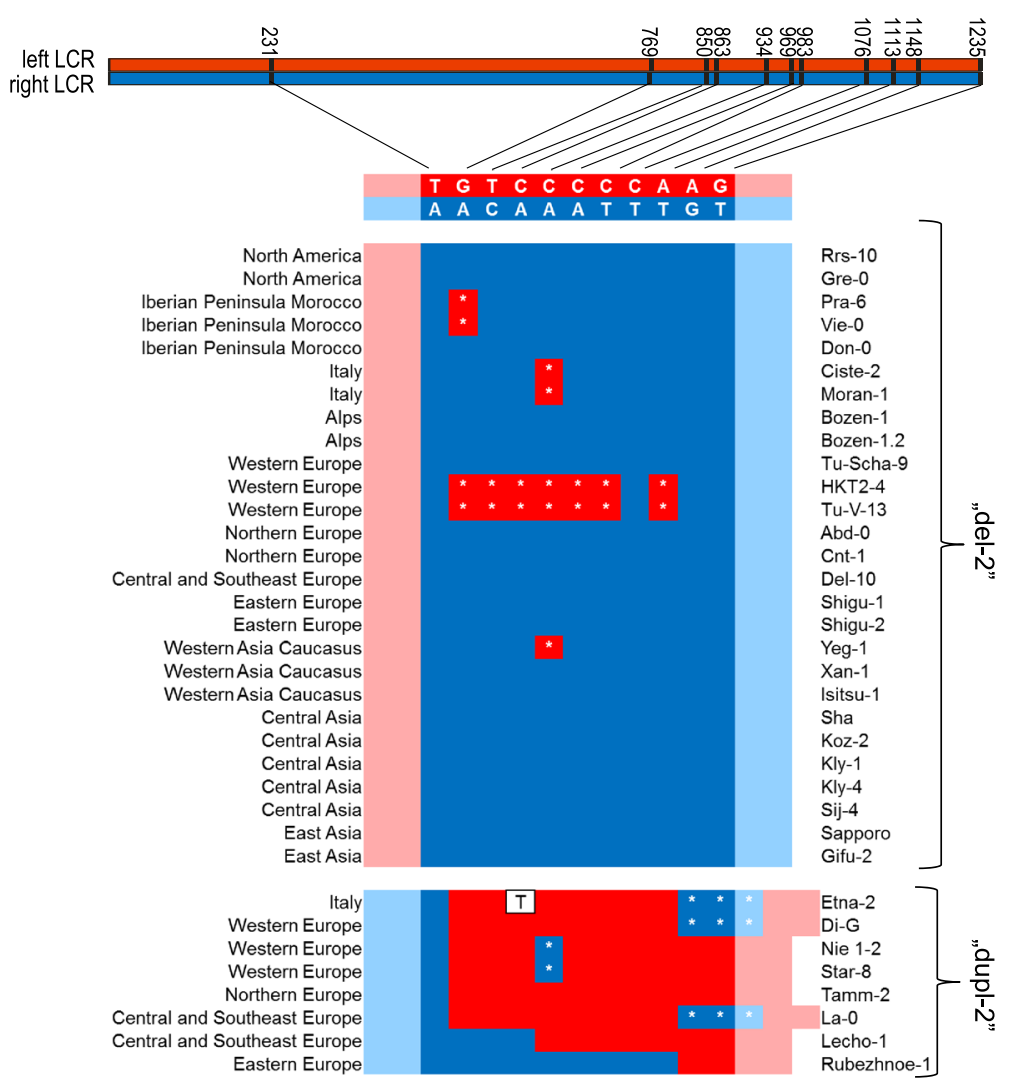

Fig. 8 Sequence analysis of chromosome breakpoints in accessions with "del-2" and "dupl-2" genotypes. The 1238-bp long LCRs differ in 11 positions. Left and right LCR are colored in red and blue, respectively. The variable sites which differentiate both LCRs are indicated - they accumulate in the right half of the repeat. The upstream and downstream sequences directly adjacent to each LCR are marked in respective but less intensive color. White asterisks indicate gene conversion events. Single nucleotide substitution found in the variable site in one accession is presented on white background

recently estimated that $\mathrm{mCNVs}$ contribute about 7 times more to the gene dosage variation than much more abundant simple biallelic CNVs [38].

The complex $\mathrm{mCNV}$ characterized in our study spans 3 protein-coding genes, $\mathrm{MSH} 2$, encoding a key component of the mismatch repair pathway, and AT3G18530 and AT3G18535, for which little functional information exists. The experimental data obtained in the present study which involved 189 Arabidopsis accessions indicated that $53.4 \%$ of them lost the DNA region harboring the latter genes. According to our analysis of WGS data available for 1,135 accessions form 1001 Genomes collection, this rate is even higher and over $60 \%$ of accessions might actually harbor the "del-2" genotype. Therefore, AT3G18530 and AT3G18535 genes are not strictly essential for plant growth. The "del-2" genotype is spread across divergent habitats and geographical locations and the results of the analysis of surrounding haplotype SNPs suggested that the deletions occurred independently multiple times. Remarkably, 17 out of 25 relicts (accessions that inhabit ancestral habitat) [44], including one extremely divergent accession from the
Cape Verde Islands likely possess "del-2" genotype. For two of them, Don-0 and Ped-0, we provided experimental verification. We also detected reciprocal duplications of the block of AT3G18530-AT3G18535 genes ("dupl-2" genotype). The analysis of chromosome breakpoints performed in one-fourth of the accessions with "del-2" genotype (including relict Don-0) and in all but one accession with the "dupl-2" genotype (including Etna-2, an admixed accession of relict and non-relict) revealed that the events underlying structural rearrangements recurrently involved homology-based recombination between segmental duplications flanking the block of these two genes. In humans, the deletions/duplications between directly oriented LCRs show twofold genomewide enrichment [53]. However, unlike diseaseassociated CNVs, which have been extensively studied in humans, the CNV described in the present study might represent a region under lower selective pressure (although this information needs further verification). Nevertheless, the precise localization of a genomic region highly prone to NAHR in the model plant Arabidopsis suggests some interesting possibilities regarding 
molecular studies on this phenomenon or targeted gene duplication.

NAHR is acknowledged to be the key mechanism leading to the formation of recurrent CNVs in animals and humans $[24,54,55]$ and our molecular analysis (detection of reciprocal genotypes, breakpoints at LCR regions with no additional inserted sequence, sequence conversion events, random geographic distribution) provided strong evidence that AT3G18530-AT3G18535 CNV was mediated by this mechanism, as well. It should be acknowledged, however, that it does not exclude the possibility that additional nonhomologous or microhomology-driven steps were involved in formation of the complex duplications at this site as well (exemplified by Etna-2, La-0 and Di-G accessions). In fact, extensive analysis of complex structural variations present in the human genomes revealed that in many cases, the template-switching mechanisms might contribute to their structural complexity [ 55 , 56]. The third gene investigated in the present study, which also lies within $\mathrm{mCNV}$, is $\mathrm{MSH} 2$. Although the previously described CNV_610 region [29] only partially covered the MSH2 locus, the MLPA data obtained in the present study show that this duplication is larger (presumably affecting the entire gene). We observed the striking association of the $M S H 2$ copy number with the copy numbers of AT3G18530 and AT3G18535. The MSH2 duplication was not combined with the duplication of the remaining genes in only one case. In 11 accessions, all three of genes were duplicated, and in 7 accessions, the gene copy numbers were identical according to the ddPCR analysis. In 2 accessions, the $M S H 2$ copy number was lower (by 2 copies) and in 2 other accessions the MSH2 copy number was higher (by 2 copies) than AT3G18530-AT3G18535 (Additional file 2: Table S1). Additionally, high copy number alleles (8 copies and more) were exclusively observed among accessions with "dupl-3" genotypes. This finding suggests that the region harboring all three genes underwent duplications, likely involving molecular events different from those leading to the generation of the "dupl-2" and "del-2" genotypes. However, at present, we did not attempt to dissect these mechanisms or the chromosome breakpoints in accessions with the "dupl-3" genotype.

$\mathrm{CNV}$ may affect gene dosage and consequent phenotype [16]. MSH2 is a conserved gene, essential for maintaining genome stability and preventing recombination events between non-identical sequences [57]. Mutations in this gene lead to microsatellite instability [58]. Moreover, Atmsh2-1 knockout mutant lines rapidly accumulate mutations and show abnormalities in morphology and development, fertility, germination efficiency, seed/silique development, and seed set when propagated for 5 generations [59]. Consistent with these findings, none of the Arabidopsis accessions analyzed in the present study harbored $\mathrm{MSH} 2$ deletions.
Although evaluating the effect of the $\mathrm{MSH} 2$ gene duplication at the transcription level was beyond the scope of the present study, we aim to highlight the potential impact of this variation. Future studies of accessions naturally varying according to MSH2 copy number might enhance the functional characterization of the mismatch repair mechanisms in plants.

One issue concerning WGS-based CNV discovery is that different bioinformatics approaches might produce results (CNV lists) showing little overlap [1]. Therefore, the routine verification of new CNVs and the examination of the frequency of these changes in plant populations are needed before this new knowledge can be broadly applied to genome-wide association and agrigenomics studies. In humans, where CNV-genotyping is a routine task $[60,61]$, including the availability of commercial assays for some disease-related genes, multiple gene-overlapping CNVs have been studied in detail. No such standardized protocols exist for plants and the available CNV data are not abundant or preliminary. Previous reports regarding variation of Arabidopsis MSH2, AT3G18530 and AT3G18535 genes include CNV_610 and CNV_611 inferred by Cao et al. in Eurasian populations (Additional file 1: Figure S1) [29] and variation analysis among Swedish accessions, where two neighboring 3-kb duplicated regions were detected (variant Chr3:6375000 was present in FäL 1, Fäb-2, Tamm-2, TFÄ 06 and Västervik; variant Chr3:6372000 was present in FäL 1 and Fäb-2 only) [62]. Here we provided molecular evidence (MLPA, ddPCR and Sanger-based) that Tamm-2 harbors duplication of both AT3G18530 and AT3G18535 genes. Overall, the comprehensive information about a $\mathrm{CNV}$ status of these loci in Arabidopsis population may serve as a "gold standard" to validate genomewide tools for variant calling from WGS data.

In the present study, we compared two molecular approaches towards locus-specific $\mathrm{CNV}$ analysis that were optimized for usage in Arabidopsis: MLPA and ddPCR. We also designed sets of control probes and primers, localized in experimentally validated copy number stable regions, for genotyping any $\mathrm{CNV}$ of interest in Arabidopsis. MLPA is considered a gold standard in the molecular diagnosis of human diseases resulting from DNA copy number alterations [60]. Furthermore, MLPA has multiplexing ability and is cost-efficient when the analysis of hundreds or thousands of DNA samples is needed. Unlike ddPCR, which typically requires some optimization steps, MLPA is consistently performed under the same uniform conditions. However, the initial high cost of probe synthesis makes this approach suboptimal for CNV analysis in a smaller number of samples. In such cases, ddPCR would be much more time and cost-efficient, as this approach requires only standard PCR primers and is a one-step method, exhibiting exceptional sensitivity with less possibility for the introduction of errors [36, 63]. 
The discrete genotyping of $\mathrm{mCNVs}$ is a challenging task, and typically, the precise copy numbers could only be estimated for a lower number of copies $(<6)$ [64-66]. Previously, we presented the robustness of the MLPA approach for the high-resolution genotyping of several disease-related $\mathrm{mCNVs}$ in humans, accurately genotyping integer copy numbers of up to 8 gene copies [41, 43]. More recently, ddPCR has been shown to provide accurate copy number assessments for $\mathrm{mCNV}$ in human populations, ranging from 0-15 copies [38]. Through direct comparison of the two approaches, we showed that ddPCR outperformed MLPA in discriminating high copy numbers. However, both methods produced highly consistent results and facilitated accurate copy number assessment across a common range of diploid copy numbers ( $0-8$ copies). Moreover, both approaches are easy to apply for the systematic genotyping of gene CNV in plants and the validation of WGS data.

\section{Conclusions}

Here we presented the first detailed, population-scale analysis of a complex CNV of a particular locus in Arabidopsis. We dissected the structure of a complex mCNV, spanning the MSH2, AT3G18530 and AT3G18535 genes in Arabidopsis genome, and evaluated the range and ecogeographical distribution of the copy numbers of these genes. The presented data provided insight into the mechanisms and factors driving CNV evolution in Arabidopsis and allowed us to create a model of recurrent duplications/deletions of AT3G18530 and AT3G18535 through homologous recombination between long repeats, flanking these genes. Our comprehensive case study provides foundation information for further analyses of CNV evolution in Arabidopsis and other plants, and their possible use in plant breeding. We also successfully applied two experimental approaches (MLPA and ddPCR) for CNV analysis in this plant. We directly compared the two techniques and showed their consistency in detecting DNA copy numbers up to 8 . While MLPA is highly suitable for multiplexed analysis of several loci in a large number of samples, ddPCR should be the method of choice for discriminating loci with high copy numbers.

\section{Methods}

\section{Plant materials}

Arabidopsis seeds were obtained from NASC The European Arabidopsis Stock Centre. The seeds were surface-sterilized, vernalized for 3 days and subsequently planted on Jiffy pellets in ARASYSTEM containers (BETATECH). The plants were grown for 3 weeks in a growth chamber under long day conditions (16-h light; 8h dark; $22{ }^{\circ} \mathrm{C} / 18{ }^{\circ} \mathrm{C}, 70 \%$ humidity), nourished with Murashige \& Skoog medium, 0.5x (Serva). The leaves were frozen in liquid nitrogen and stored at $-80{ }^{\circ} \mathrm{C}$. Genomic DNA was extracted using the DNeasy Plant Mini Kit
(Qiagen), followed by qualitative and quantitative evaluation on a Nanodrop 2000 spectrophotometer (Thermo Scientific) and with standard gel electrophoresis.

\section{MLPA probe set design and data analysis}

The synthetic oligonucleotide half probes were designed as previously described [40, 41] and purchased (Integrated DNA Technologies). Each half probe comprised a target-specific sequence (23-29 nt each), the stuffer sequence of variable length and a universal primer sequence (Additional file 1: Table S2). Preferentially, target-specific sequences were localized in gene exons in SNP-free regions (based on a SNP map available for MPICao2010 data set [67]. The MLPA assays were performed using the SALSA MLPA Reagent Kit (MRC-Holland) according to the manufacturer's guidelines, starting with $100 \mathrm{ng}$ of genomic DNA and $1 \mathrm{pM}$ of each half probe per reaction. The amplification products were separated through capillary electrophoresis on an ABI Prism 3130XL Genetic Analyzer (Applied Biosystems). The peak heights (signal intensities) were retrieved from the electropherograms using GeneMarker v.2.4.0 (SoftGenetics). For each sample, the peak heights were normalized to the average of the control probes. The low variation of the normalized values observed for probes ctrl1 to ctrl5 (SD <10\%) was used to verify the high quality of all MLPA assays. To facilitate data comparison across the probes and samples, the data are presented as a ratio to Col-0 accession. In 179 samples, the ratios for all control probes were between 0.8 and 1.2 (expected: 1.0) (Additional file 2: Table S1). In the remaining 10 samples, the ratio for only one control probe (ctrl4 or ctrl5, which generated DNA amplicons of the most extreme sizes in the Ath_CNV610-611_MLPA assay) showed only a small deviation from this range (min. observed ratio was 0.67 and max. was 1.38). Based on this analysis, the threshold values for deletion and duplication were set at 0.5 and 1.5 , respectively.

\section{Droplet digital PCR assay design and analysis}

The optimal sample concentration and primer annealing temperatures were evaluated in a series of test assays (0.05-50 ng DNA/well range and 56-60 ${ }^{\circ} \mathrm{C}$ temperature range tested, respectively, see Additional file 1: Figures S12 and S13). The final gene copy number assays were performed in $20-\mu \mathrm{l}$ reactions containing $1 \times$ EvaGreen ddPCR Supermix (Bio-Rad), $200 \mathrm{nM}$ gene specific primers (Additional file 1: Figure S2 and Table S3) and XbaI-digested DNA samples $(2.5 \mathrm{ng})$. All XbaI restriction sites were located outside the predicted amplicons. Each reaction was mixed with $70 \mu \mathrm{l}$ of Droplet Generation Oil (BioRad), partitioned into approximately 18,000 droplets in a QX200 Droplet Generator (Bio-Rad), transferred 
to 96-well plates (Eppendorf) and sealed. The PCRs were performed using a C1000 Touch Thermal Cycler (Bio-Rad) with the following cycling conditions: $1 \times$ $\left(95{ }^{\circ} \mathrm{C}\right.$ for $\left.5 \mathrm{~min}\right), 40 \times\left(95{ }^{\circ} \mathrm{C}\right.$ for $30 \mathrm{~s}, 57{ }^{\circ} \mathrm{C}$ for $30 \mathrm{~s}, 72{ }^{\circ} \mathrm{C}$ for $\left.45 \mathrm{~s}\right), 1 \times\left(4{ }^{\circ} \mathrm{C}\right.$ for $5 \mathrm{~min}, 90{ }^{\circ} \mathrm{C}$ for $5 \mathrm{~min}$ ) with $2{ }^{\circ} \mathrm{C} / \mathrm{s}$ ramp rate. Immediately following end-point amplification, the fluorescence intensity of the individual droplets was measured using the QX200 Droplet Reader (Bio-Rad). The data analysis was performed using QuantaSoft droplet reader software (Bio-Rad). Positive and negative droplet populations were automatically detected. The template copy numbers [copies/ $\mu \mathrm{l}$ PCR] were calculated using Poisson statistics and background-corrected based on the notemplate control data. The absolute DNA copy numbers were subsequently obtained through within-sample normalization against data for the DCL1 control gene. In all samples assayed, the calculated diploid copy number of the non-variable $H D A 15$ and $B R C 1$ genes flanking the analyzed $\mathrm{CNV}$ region was 2 , confirming the accuracy of this normalization approach.

\section{CNV breakpoint detection and analysis}

The genomic region covered through the Ath_CNV610611_MLPA assay was divided into non-overlapping 1000bp windows and subsequently BLASTN-searched against each other. The regions of significant similarity were further individually inspected and merged or extended to obtain final genomic coordinates of repeats. In accessions with the "del-2" CNV pattern, the regions of chromosome breakpoints were subsequently amplified using the following primers: forward: 5'-CCTAGAGCAGGAGTCG CAAG-3' and reverse: 5'-CGCTTAAGTTAAGGAGA TTTGACAACACCACAT-3.' In accessions with the "dupl-2" CNV pattern, the regions of chromosome breakpoints were amplified using the following primers: left: 5'GTGGGGGAGTTTGTGTCTCA-3' and right: 5'- GTGG ATTAAGGCGAATTCGACGACGAGATC-3.' The amplifications were performed using PrimeSTAR GXL DNA Polymerase (Takara Bio), and the PCR products were resolved through agarose electrophoresis. For DNA sequencing, the PCR products were purified using the Clean \& Concentrator Kit (Zymo) and sequenced on an ABI Prism 3130XL Genetic Analyzer (Applied Biosystems) using the Big Dye Terminator v.1.1 Cycle Sequencing Kit (Applied Biosystems).

\section{WGS-based variation analysis in 1001 genomes accessions}

A list of SNPs in 20-kb genomic regions flanking the analyzed CNVs from each side (Chr3:6348000..6368000 and Chr3:6380000..6400000) was obtained from the 1001 Genomes Project server [67]. Out of 189 accessions used in this study the SNP data were available for
153 accessions. Additionally, Col-0 accession was included, with reference alleles in all positions. The positions were filtered to include only SNPs that were biallelic among 1,135 genomes. Subsequently, only SNPs of at least $10 \%$ frequency among the subset of 154 analyzed accessions and with less than $20 \%$ missing values per position were retained for the analysis. The SNPs were concatenated, aligned and imported to SplitsTree (version 4.14.4) in Nexus format (ready-to-use data file is included as Additional file 3). The phylogenetic network was then constructed using NeighborNet method [45]. $\mathrm{LD}$ analysis and $\mathrm{R}^{2}$ calculation was performed on the same data with the use of LDPlotter tool [68]. For the evaluation of "del-2" genotype prevalence, we obtained the sequences of AT3G18530-AT3G18535 loci for 1,135 accessions using the Pseudogenome tool available in 1001 Genomes server.

\section{Additional files}

\begin{abstract}
Additional file 1: Figure S1. Distribution of DNA copy number in regions covered by CNV_610 and CNV_611 in 80 natural accessions of Arabidopsis (MPICao2010 set). Figure S2. A schematic map of Arabidopsis genes covered by AthMSH2-MLPA assay. Figure S3. Exemplar electropherograms of AthMSH2-MLPA assay results. Figure S4. Pairwise correlation of MLPA signals obtained with probes mlpaB-mlpaG in AthMSH2-MLPA genotyping assay of Arabidopsis populations. Figure S5. Nonhierarchical phylogenetic network of a subset of 154 accessions based on 20-kb regions flanking the CNVs and its relation to the genetic groups defined by 1001 Genomes Consortium. Figure S6. Linkage disequilibrium (LD) at genomic regions surrounding the investigated CNVs. Figure S7. Rate of missing calls at AT3G18530-AT3G18535 loci in pseudogenome sequences of 1135 Arabidopsis accessions. Figure S8. The sequence composition of the left and right breakpoints in accessions with "del-2" and "dupl-2" genotypes. Figure S9. Sequence alignment of CNV breakpoints in accessions with "del-2" genotype. Figure S10. Sequence alignment of CNV breakpoints in accessions with simple "dupl-2" genotype. Figure S11. Sequence alignment of CNV breakpoints in accessions with "dupl-2" genotype harboring extended duplication, that involves also the $3^{\prime}$ flank of the right LCR. Figure S12. Optimization of genomic DNA template input for ddPCR. Figure S13. Optimization of primer annealing temperatures for ddPCR. Table S2. Sequences of MLPA probes. Table S3. Gene specific primers used for ddPCR assays. (PDF 1563 kb)

Additional file 2: Table S1. List of Arabidopsis accessions and gene copy numbers determined in MLPA- and ddPCR-based study. (XLSX 81 kb)
\end{abstract}

Additional file 3: Matrix data file with SNP data for 154 accessions, used for the construction of phylogenetic network. (TXT 16 kb)

\section{Abbreviations \\ CNV: Copy number variation; ddPCR: Droplet digital PCR; dHj: Double Holliday junction; DSB: Double strand break; EPSP: 5-enolpyruvylshikimate 3- phosphate; LCR: Low copy repeat; LD: Linkage disequilibrium; mCNV: Multiallelic CNV; MLPA: Multiplex ligation-dependent probe amplification; NAHR: Non-allelic homologous recombination; SCN: Soybean cyst nematode; WGS: Whole-genome sequencing}

\section{Acknowledgements}

We are grateful to Bio-Rad Polska for providing access to the QX200 instrument. We thank Anna Philips for help with analysis of vcf files.

\section{Funding}

This work has been conducted in ECBiG centre, with the support from National Centre of Science (grants 2011/01/B/NZ2/04816 and 2014/13/B/ 
NZ2/03837) and Polish Ministry of Science and Higher Education (KNOW program).

\section{Availability of data and materials}

The datasets supporting the conclusions of this article are included within the article (and its additional files). The matrix data in NEXUS format, used for the construction of phylogenetic network presented in Figure S5, are included as Additional file 3, ready to use with SplitsTree4 program [45], that is freely provided by University of Tübingen at http://www.splitstree.org/.

\section{Authors' contributions}

AZ conceived of the study, designed and oversaw all experiments; analyzed MLPA and ddPCR data, analyzed the CNV geographical distribution and duplication/deletion breakpoint sequencing data; created the model of NAHR-based duplications and deletions; prepared figures and drafted the manuscript. ASCz prepared DNA samples, performed MLPA and ddPCR assays, helped to analyze respective data and prepared input data for SNP analysis. MSz carried out PCR assays and Sanger sequencing experiments. PK performed SNP analysis and helped to draft the manuscript. MF conceived of the study, interpreted the data, oversaw the research and finalized the manuscript. All authors read and approved the final manuscript.

\section{Competing interests}

The authors declare that they have no competing interests.

\section{Consent for publication}

Not applicable.

\section{Ethics approva}

Not applicable.

\section{Received: 10 June 2016 Accepted: 27 October 2016}

Published online: 08 November 2016

\section{References}

1. Alkan C, Coe BP, Eichler EE. Genome structural variation discovery and genotyping. Nat Rev Genet. 2011;12:363-76.

2. Chen W-K, Swartz JD, Rush LJ, Alvarez CE. Mapping DNA structural variation in dogs. Genome Res. 2009;19:500-9.

3. Springer NM, Ying K, Fu Y, Ji T, Yeh C-T, Jia Y, et al. Maize inbreds exhibit high levels of copy number variation (CNV) and presence/absence variation (PAV) in genome content. PLoS Genet. 2009;5:e1000734.

4. Sudmant PH, Kitzman JO, Antonacci F, Alkan C, Malig M, Tsalenko A, et al. Diversity of human copy number variation and multicopy genes. Science. 2010;330:641-6.

5. Haun WJ, Hyten DL, Xu WW, Gerhardt DJ, Albert TJ, Richmond T, et al. The composition and origins of genomic variation among individuals of the soybean reference cultivar Williams 82. Plant Physiol. 2011;155:645-55.

6. Hou Y, Liu GE, Bickhart DM, Cardone MF, Wang K, Kim E-S, et al. Genomic characteristics of cattle copy number variations. BMC Genomics. 2011;12:127.

7. McHale LK, Haun WJ, Xu WW, Bhaskar PB, Anderson JE, Hyten DL, et al. Structural variants in the soybean genome localize to clusters of biotic stress-response genes. Plant Physiol. 2012;159:1295-308.

8. Gonzalez E, Kulkarni H, Bolivar H, Mangano A, Sanchez R, Catano G, et al. The influence of CCL3L1 gene-containing segmental duplications on HIV-1/ AIDS susceptibility. Science. 2005;307:1434-40.

9. Perry GH, Dominy NJ, Claw KG, Lee AS, Fiegler $H$, Redon $R$, et al. Diet and the evolution of human amylase gene copy number variation. Nat Genet. 2007:39:1256-60.

10. Mills RE, Walter K, Stewart C, Handsaker RE, Chen K, Alkan C, et al. Mapping copy number variation by population-scale genome sequencing. Nature. 2011;470:59-65.

11. Handsaker RE, Korn JM, Nemesh J, McCarroll SA. Discovery and genotyping of genome structural polymorphism by sequencing on a population scale. Nat Genet. 2011;43:269-76

12. Gaines TA, Zhang W, Wang D, Bukun B, Chisholm ST, Shaner DL, et al. Gene amplification confers glyphosate resistance in Amaranthus palmeri. Proc Natl Acad Sci U S A. 2010;107:1029-34

13. Cook DE, Lee TG, Guo X, Melito S, Wang K, Bayless AM, et al. Copy number variation of multiple genes at $R$ h 1 mediates nematode resistance in soybean. Science. 2012;338:1206-9.
14. Maron LG, Guimarães CT, Kirst M, Albert PS, Birchler JA, Bradbury PJ, et al. Aluminum tolerance in maize is associated with higher MATE1 gene copy number. Proc Natl Acad Sci U S A. 2013;110:5241-6.

15. Sutton T, Baumann U, Hayes J, Collins NC, Shi BJ, Schnurbusch T, et al. Boron-toxicity tolerance in barley arising from efflux transporter amplification. Science. 2007:318:1446-9.

16. Żmieńko A, Samelak A, Kozłowski P, Figlerowicz M. Copy number polymorphism in plant genomes. Theor Appl Genet. 2014;127:1-18.

17. Díaz A, Zikhali M, Turner AS, Isaac P, Laurie DA. Copy number variation affecting the Photoperiod-B1 and Vernalization-A1 genes is associated with altered flowering time in wheat (Triticum aestivum). PLoS One. 2012;7:e33234.

18. Nitcher R, Distelfeld A, Tan C, Yan L, Dubcovsky J. Increased copy number at the HVFT1 locus is associated with accelerated flowering time in barley. Mol Genet Genomics. 2013:288:261-75.

19. Loscos J, Igartua E, Contreras-Moreira B, Gracia MP, Casas AM HvFT1polymorphism and effect-survey of barley germplasm and expression analysis. Front Plant Sci. 2014;5:251

20. Schiessl S, Samans B, Hüttel B, Reinhard R, Snowdon RJ. Capturing sequence variation among flowering-time regulatory gene homologs in the allopolyploid crop species Brassica napus. Front Plant Sci. 2014;5:404.

21. Würschum T, Boeven PH, Langer SM, Longin CF, Leiser WL. Multiply to conquer: Copy number variations at Ppd-B1 and Vrn-A1 facilitate global adaptation in wheat. BMC Genet. 2015;16:96

22. Wang $Y$, Xiong $G, H u$ J, Jiang $L, Y u ~ H, X u ~ J$, et al. Copy number variation at the GL7 locus contributes to grain size diversity in rice. Nat Genet. 2015:47:944-8.

23. Cook DE, Bayless AM, Wang K, Guo X, Song Q, Jiang J, et al. Distinct Copy Number, Coding Sequence, and Locus Methylation Patterns Underlie Rhg 1Mediated Soybean Resistance to Soybean Cyst Nematode. Plant Physiol. 2014; 165:630-47.

24. Gu W, Zhang F, Lupski JR. Mechanisms for human genomic rearrangements. Pathogenetics. 2008;1:4.

25. Dittwald P, Gambin T, Szafranski P, Li J, Amato S, Divon MY, et al. NAHRmediated copy-number variants in a clinical population: mechanistic insights into both genomic disorders and Mendelizing traits. Genome Res. 2013;23:1395-409

26. Cannon SB, Mitra A, Baumgarten A, Young ND, May G. The roles of segmental and tandem gene duplication in the evolution of large gene families in Arabidopsis thaliana. BMC Plant Biol. 2004:4:10.

27. Flagel LE, Wendel JF. Gene duplication and evolutionary novelty in plants. New Phytol. 2009;183:557-64.

28. Fawcett JA, Van de Peer Y, Maere S. Significance and Biological Consequences of Polyploidization in Land Plant Evolution. In: Greilhuber J, Dolezel J, Wendel JF, editors. Plant Genome Diversity Volume 2. Vienna: Springer; 2013. p. 277-93.

29. Cao J, Schneeberger K, Ossowski S, Günther T, Bender S, Fitz J, et al. Wholegenome sequencing of multiple Arabidopsis thaliana populations. Nat Genet. 2011:43:956-63.

30. Culligan KM, Hays JB. Arabidopsis MutS homologs-AtMSH2, AtMSH3, AtMSH6, and a novel AtMSH7-form three distinct protein heterodimers with different specificities for mismatched DNA. Plant Cell. 2000;12:991-1002

31. Emmanuel E, Yehuda E, Melamed-Bessudo C, Avivi-Ragolsky N, Levy AA. The role of AtMSH2 in homologous recombination in Arabidopsis thaliana. EMBO Rep. 2006;7:100-5.

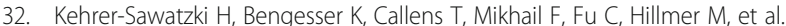
Identification of large NF1 duplications reciprocal to NAHR-mediated type-1 NF1 deletions. Hum Mutat. 2014;35:1469-75.

33. Bengesser K, Vogt J, Mussotter T, Mautner V-F, Messiaen L, Cooper DN, et al. Analysis of crossover breakpoints yields new insights into the nature of the gene conversion events associated with large NF1 deletions mediated by nonallelic homologous recombination. Hum Mutat. 2014;35:215-26.

34. Stankiewicz $P$, Lupski JR. Structural variation in the human genome and its role in disease. Annu Rev Med. 2010;61:437-55.

35. Kozlowski P, Jasinska AJ, Kwiatkowski DJ. New applications and developments in the use of multiplex ligation-dependent probe amplification. Electrophoresis. 2008;29:4627-36.

36. Hindson BJ, Ness KD, Masquelier DA, Belgrader P, Heredia NJ, Makarewicz AJ, et al. High-throughput droplet digital PCR system for absolute quantitation of DNA copy number. Anal Chem. 2011;83:8604-10. 
37. Miotke L, Lau BT, Rumma RT, Ji HP. High sensitivity detection and quantitation of DNA copy number and single nucleotide variants with single color droplet digital PCR. Anal Chem. 2014;86:2618-24.

38. Handsaker RE, Van Doren V, Berman JR, Genovese G, Kashin S, Boettger LM, et al. Large multiallelic copy number variations in humans. Nat Genet. 2015:47:296-303.

39. Weigel D, Mott R. The 1001 genomes project for Arabidopsis thaliana. Genome Biol. 2009;10(5):107. doi:10.1186/gb-2009-10-5-107.

40. Marcinkowska M, Wong K-K, Kwiatkowski DJ, Kozlowski P. Design and generation of MLPA probe sets for combined copy number and smallmutation analysis of human genes: EGFR as an example. ScientificWorldJournal. 2010;10:2003-18.

41. Marcinkowska-Swojak M, Uszczynska B, Figlerowicz M, Kozlowski P. An MLPA-based strategy for discrete CNV genotyping: CNV-miRNAs as an example. Hum Mutat. 2013;34:763-73.

42. Barbaro $M$, Kotajärvi $M$, Harper $P$, Floderus $Y$. Partial protoporphyrinogen oxidase (PPOX) gene deletions, due to different Alu-mediated mechanisms, identified by MLPA analysis in patients with variegate porphyria. Orphanet J Rare Dis. 2013;8:13.

43. Marcinkowska-Swojak M, Klonowska K, Figlerowicz M, Kozlowski P. An MLPA-based approach for high-resolution genotyping of disease-related multi-allelic CNVs. Gene. 2014;546:257-62.

44. 1001 Genomes Consortium. 1,135 Genomes Reveal the Global Pattern of Polymorphism in Arabidopsis thaliana. Cell. 2016;166:481-91.

45. Bryant D, Moulton V. Neighbor-Net: An Agglomerative Method for the Construction of Phylogenetic Networks. Mol Biol Evol. 2004;21:255-65.

46. Sharbel TF, Haubold B, Mitchell-Olds T. Genetic isolation by distance in Arabidopsis thaliana: biogeography and postglacial colonization of Europe. Mol Ecol. 2000;9:2109-18.

47. Carvalho CM, Pehlivan D, Ramocki MB, Fang P, Alleva B, Franco LM, et al Replicative mechanisms for CNV formation are error prone. Nat Genet. 2013:45:1319-26.

48. Chia J-M, Song C, Bradbury PJ, Costich D, de Leon N, Doebley J, et al. Maize HapMap2 identifies extant variation from a genome in flux. Nat Genet. 2012;44:803-7

49. Xu X, Liu X, Ge S, Jensen JD, Hu F, Li X, et al. Resequencing 50 accessions of cultivated and wild rice yields markers for identifying agronomically important genes. Nat Biotechnol. 2012;30:105-11.

50. Boocock J, Chagné D, Merriman TR, Black MA. The distribution and impact of common copy-number variation in the genome of the domesticated apple, Malus x domestica Borkh. BMC Genomics. 2015;16:848.

51. Tan S, Zhong Y, Hou H, Yang S, Tian D. Variation of presence/absence genes among Arabidopsis populations. BMC Evol Biol. 2012;12:86.

52. Olsson LM, Holmdahl R. Copy number variation in autoimmunityimportance hidden in complexity? Eur J Immunol. 2012;42:1969-76.

53. Li J, Harris RA, Cheung SW, Coarfa C, Jeong M, Goodell MA, et al. Genomic hypomethylation in the human germline associates with selective structural mutability in the human genome. PLoS Genet. 2012;8:e1002692

54. Lu P, Han X, Qi J, Yang J, Wijeratne AJ, Li T, et al. Analysis of Arabidopsis genome-wide variations before and after meiosis and meiotic recombination by resequencing Landsberg erecta and all four products of a single meiosis. Genome Res. 2011;22:508-18.

55. Liu P, Carvalho CM, Hastings PJ, Lupski JR. Mechanisms for recurrent and complex human genomic rearrangements. Curr Opin Genet Dev. 2012;22:211-20.

56. Sudmant PH, Rausch T, Gardner EJ, Handsaker RE, Abyzov A, Huddleston J, et al. An integrated map of structural variation in 2,504 human genomes. Nature. 2015;526:75-81.

57. Spampinato CP, Gomez RL, Galles C, Lario LD. From bacteria to plants: A compendium of mismatch repair assays. Mutat Res. 2009;682:110-28.

58. Leonard JM, Bollmann SR, Hays JB. Reduction of stability of Arabidopsis genomic and transgenic DNA-repeat sequences (microsatellites) by inactivation of AtMSH2 mismatch-repair function. Plant Physiol. 2003;133:328-38.

59. Hoffman PD, Leonard JM, Lindberg GE, Bollmann SR, Hays JB. Rapid accumulation of mutations during seed-to-seed propagation of mismatchrepair-defective Arabidopsis. Genes Dev. 2004;18:2676-85.

60. Stuppia L, Antonucci I, Palka G, Gatta V. Use of the MLPA assay in the molecular diagnosis of gene copy number alterations in human genetic diseases. Int J Mol Sci. 2012;13:3245-76.
61. Cantsilieris S, Baird PN, White SJ. Molecular methods for genotyping complex copy number polymorphisms. Genomics. 2013;101:86-93.

62. Long Q, Rabanal FA, Meng D, Huber CD, Farlow A, Platzer A, et al. Massive genomic variation and strong selection in Arabidopsis thaliana lines from Sweden. Nat Genet. 2013;45:884-90.

63. Pinheiro LB, Coleman VA, Hindson CM, Herrmann J, Hindson BJ, Bhat S, et al. Evaluation of a droplet digital polymerase chain reaction format for DNA copy number quantification. Anal Chem. 2012;84:1003-11.

64. McCarroll SA, Kuruvilla FG, Korn JM, Cawley S, Nemesh J, Wysoker A, et al. Integrated detection and population-genetic analysis of SNPs and copy number variation. Nat Genet. 2008:40:1166-74.

65. Conrad DF, Pinto D, Redon R, Feuk L, Gokcumen O, Zhang Y, et al. Origins and functional impact of copy number variation in the human genome. Nature. 2010;464:704-12.

66. Cantsilieris S, Western PS, Baird PN, White SJ. Technical considerations for genotyping multi-allelic copy number variation (CNV) in regions of segmental duplication. BMC Genomics. 2014;15:329.

67. 1001 Genomes Project. http://1001genomes.org/. Accessed 25 Aug 2016

68. LDPlotter. https://regepi.bwh.harvard.edu/IIPGA2/Bioinformatics. Accessed 10 Dec 2015

\section{Submit your next manuscript to BioMed Central and we will help you at every step:}

- We accept pre-submission inquiries

- Our selector tool helps you to find the most relevant journal

- We provide round the clock customer support

- Convenient online submission

- Thorough peer review

- Inclusion in PubMed and all major indexing services

- Maximum visibility for your research

Submit your manuscript at www.biomedcentral.com/submit
Biomed Central 